\title{
Air in the Heart: A Reason to Panic?
}

\author{
Anurag Mehta, $\mathrm{MD}^{7}$, Ananya Hooda, $\mathrm{MBBS}^{2}$, and Arjun Gupta, MD ${ }^{7}$ \\ 'University of Texas Southwestern Medical Centre, Dallas, TX, USA; ${ }^{2}$ S.G.T. Medical College, Hospital and Research Institute, Gurgaon, India.
}

KEYWORDS: thromboembolism; clinical images; patient safety.

J Gen Intern Med 31(8):971-2

DOI: $10.1007 / \mathrm{s} 11606-016-3637-\mathrm{y}$

(C) Society of General Internal Medicine 2016

\section{CASE}

A 37-year-old-man presented with abdominal pain. Examination was benign and laboratory studies were unremarkable. Intravenous access was established with difficulty after several attempts. Abdominal computed tomography (CT) scan revealed no acute abnormality; however, chest images revealed air in the right atrium, right ventricle and pulmonary artery (Figs. 1 and 2,2). The patient denied chest discomfort or respiratory distress. He was positioned in the left lateral, Trendelenburg position overnight and high-flow oxygen was administered. Abdominal pain resolved with conservative management and was attributed to gastroesophageal reflux.

Air embolism is a potentially catastrophic event associated with trauma, surgery, diving, mechanical ventilation and seemingly benign vascular interventions such as in our patient. ${ }^{1}$ It is suspected when patients with the above risk factors present with respiratory distress or circulatory failure (dyspnea, wheezing, chest pain, shock) or develop end-organ damage (arterial embolism). Diagnosis is made when air is detected in the circulation on $\mathrm{CT} /$ magnetic resonance imaging (MRI) scans of the chest/abdomen. Echocardiography and transcranial doppler may detect air in the heart and brain, respectively. Nonsignificant cases of venous air embolism are usually clinically silent and self-resolving with diffusion into the alveolar space, but larger ones can be fatal. Diffusion can be aided by high flow oxygen, ${ }^{2}$ and positioning the patient can prevent embolism migration. ${ }^{3}$

Received November 12, 2015

Revised January 12, 2016

Accepted February 11, 2016

Published online February 22, 2016
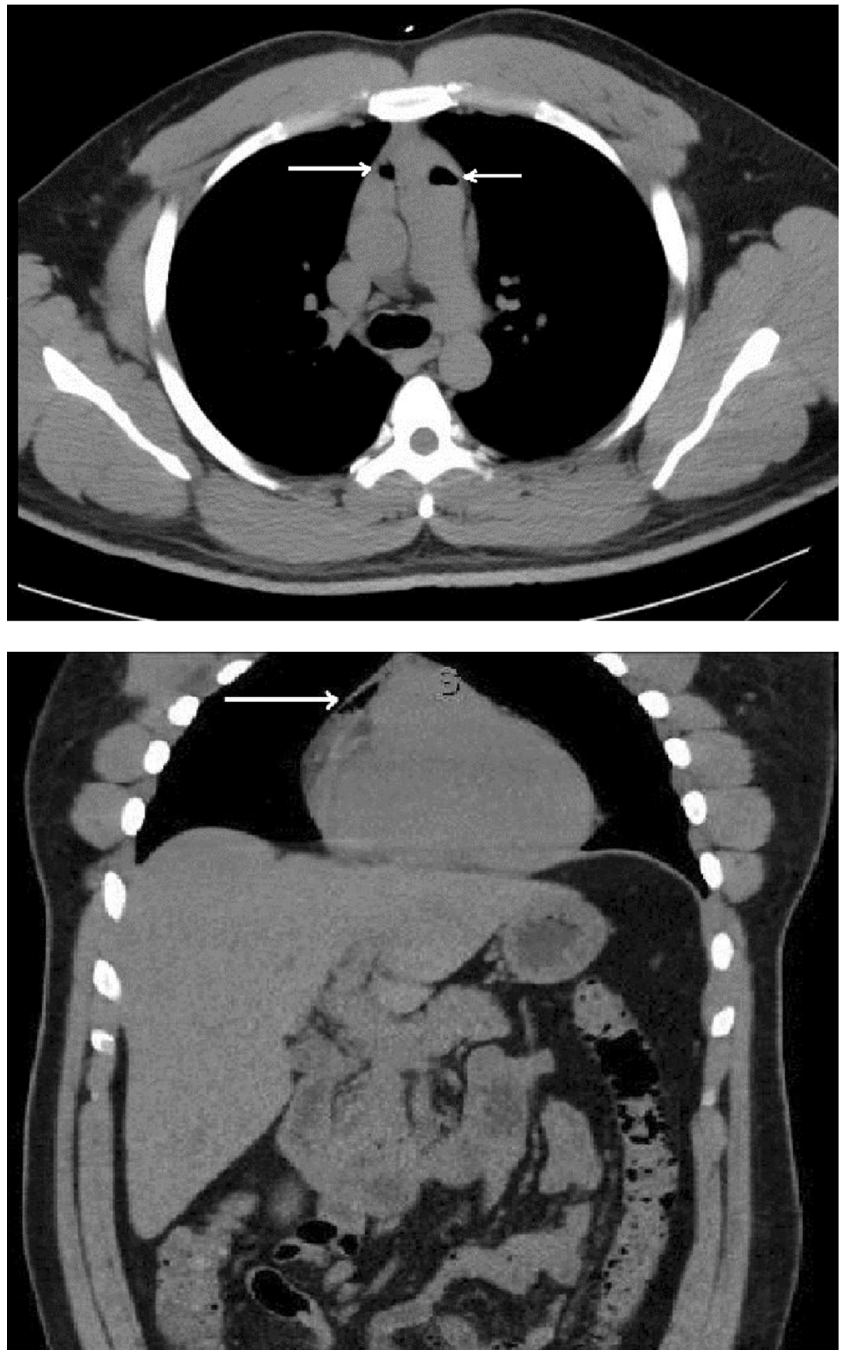

Figures 1 and 2. Computed tomography scan of the chest demonstrating air (white arrows) in the right ventricle and pulmonary artery (Figs. 1 and 2) and the right atrium (Fig. 2)

Acknowledgements: All authors have had access to all data in the study, read and approved submission of the letter, and the findings have not been presented previously in any conference or published in print or electronic format. There are no funding sources, internal or external.

Corresponding Author: Arjun Gupta, MD; University of Texas Southwestern Medical Centre, 5323 Harry Hines Blvd, Dallas, TX 75390-9047, USA (e-mail: guptaarjun90@gmail.com). 


\section{Compliance with Ethical Standards:}

Conflict of Interest: The authors declare that they do not have a conflict of interest.

\section{REFERENCES}

1. Woodring JH, Fried AM. Nonfatal venous air embolism after contrastenhanced CT. Radiology. 1988;167(2):405-407.
2. Mirski MA, Lele AV, Fitzsimmons L, Toung TJ. Diagnosis and treatment of vascular air embolism. Anesthesiology. 2007;106(1):164-177.

3. Jorens PG, Van Marck E, Snoeckx A, Parizel PM. Nonthrombotic pulmonary embolism. Eur Respir J. 2009;34(2):452-474. 